\begin{tabular}{|c|l|}
\hline Title & Osteonecrosis and panniculitis as life threatening signs \\
\hline Author(s) & Kuwatani, Masaki; Kawakami, Hiroshi; Y amada, Y osuke \\
\hline Citation & $\begin{array}{l}\text { Clinical Gastroenterology and Hepatology, 8(5), e52-e53 } \\
\text { https://doi.org/40.1016/.cgh.2009.12.026 }\end{array}$ \\
\hline Issue Date & 2010-05 \\
\hline Doc URL & http://hdl.handle.net/2115/61448 \\
\hline Rights & $\begin{array}{l}\text { O2010. This manuscript version is made available under the CC-BY-NC-ND 4.0 license } \\
\text { http://creativecommons.org/icenses/by-nc-nd/4.0/ }\end{array}$ \\
\hline Rights(URL) & http://creativecommons.org/icenses/by-nc-nd/4.0/ \\
\hline Type & PPP_syndromeCGH-revision2.pdf \\
\hline article (author version)
\end{tabular}

Instructions for use 


\section{Osteonecrosis and panniculitis as life-threatening signs}

Masaki Kuwatani ${ }^{1, *}$, Hiroshi Kawakami ${ }^{1}$, Yosuke Yamada ${ }^{2}$

${ }^{1}$ Department of Gastroenterology, Hokkaido University Graduate School of Medicine, North 15, West 7, Kita-ku, Sapporo 060-8638, Japan

${ }^{2}$ Department of Surgical pathology, Hokkaido University Hospital, North 15, West 7, Kita-ku, Sapporo 060-8638, Japan

*Address correspondence to: Dr. Masaki Kuwatani, Department of Gastroenterology, Hokkaido University Graduate School of Medicine, North 15, West 7, Kita-ku, Sapporo 060-8638, Japan E-mail: mkuwatan@med.hokudai.ac.jp (M. Kuwatani)

Telephone: +81-11-716-1161(ext. 5920). FAX: +81-11-706-7867.

No conflicts of interest exist for all authors. 
A 58-year-old male presented with polyarthritis in the bilateral wrists and ankles with elevated serum pancreatic enzymes (amylase, $4292 \mathrm{IU} / 1$; lipase, $6000 \mathrm{IU} / \mathrm{l}$ ). He had had several episodes of acute pancreatitis, and undergone cholangiojejunostomy for alcoholic chronic pancreatitis with a pseudocyst and stricture of the lower bile duct 6 years ago. Although pancreatic enzymes were temporarily normalized, roentgenograms exhibited osteonecrosis in four extremities one year after the development of polyarthritis (Figure A), and examination of a biopsy specimen of the left femur revealed intraosseous fat necrosis. CT just before admission to our department revealed slight dilatation of the main pancreatic duct and a cystic lesion adjacent to the superior mesenteric vein (SMV) in the pancreas head (Figure B). On admission, serological examinations indicated liver and renal dysfunction (elevated serum levels of AST [238 IU/1], ALT [167 IU/l], $\gamma$-GT [101 IU/1], BUN [49mg/dl], and creatinine [1.9mg/dl]) and elevated levels of pancreatic enzymes (amylase, 7966 IU/l; lipase, 12904 IU/l). Physical findings were erythema in the abdomen (Figure C) and purupura in bilateral lower extremities. Renal dysfunction rapidly exacerbated and he died 5 days after admission to our department. After informed consent from his family, autopsy was done. Gross findings of specimens of the pancreas head and the peripancreatic tissue showed a fistula between the cystic lesion in the pancreas head and SMV and exposure of debris from the cystic lesion. Microscopically, the cystic lesion was a pseudocyst connecting to the SMV, and the debris was protein plaque. The pancreas tissue surrounding the lower bile duct showed fibrous change and atrophy of the acini. In the kidneys, glomerular crescent formation was diffusely found, and a part of basement membrane was necrotic. In the skin, fibrinoid changes and infiltration of inflammatory cells were found in the walls of small arteries, and there was nodular fat necrosis with calcification (Figure D), which was also found in other organs including gastric subserosa and bone marrow. Finally, we made a diagnosis of PPP syndrome caused by pancreatic pseudocyst-portal vein fistula. A case of pancreatitis with panniculitis and polyarthrits was reported in 1968 for the first time ${ }^{1}$. PPP syndrome as terminology was suggested as a new entity in several reports, but it is a not well-known category of disease. This syndrome is frequently caused by alcoholic pancreatitis with a 
pseudocyst and occasionally by pancreatic neoplasia ${ }^{2}$. Although the mechanism remains to be determined, two possible mechanisms have been porposed: one is lysis of fat cells by high levels of pancreatic enzymes and the other is lysis of fat cells by autoimmune phenomena ${ }^{2,3}$. The main treatment for PPP syndrome is control of pancreatitis or neoplasm and drainage or preservative surgical intervention for a pseudocyst if it is present. However, the prognosis is poor, a review of the literature showing its mortality rates of $75-85 \%^{2}$. Attention should be given to the possibility of PPP syndrome if polyarthritis or osteonecrosis and panniculitis of unknown origin are detected. Delay in diagnosis of this syndrome can result in a negative chain reaction leading to death. 


\section{References}

1. Mullin GT, Caperton EM, Crespin SR, et al. Arthritis and skin lesions resembling erythema nodosum in pancreatic disease. Ann Intern Med 1968;68:75-78

2. Carasso S, Oren I, Alroy G, et al. Disseminated fat necrosis with asymptomatic pancreatitis: a case report and review of the literature. Am J Med Sci 2000;319:68-72.

3. Mustafa KN, Hadidy A, Shoumaf M, Razzuki SA. Razzuki Polyarthritis with chondronecrosis associated with osteonecrosis, panniculitis and pancreatitis. Rheumatol Int 2009; epub 
Figure A

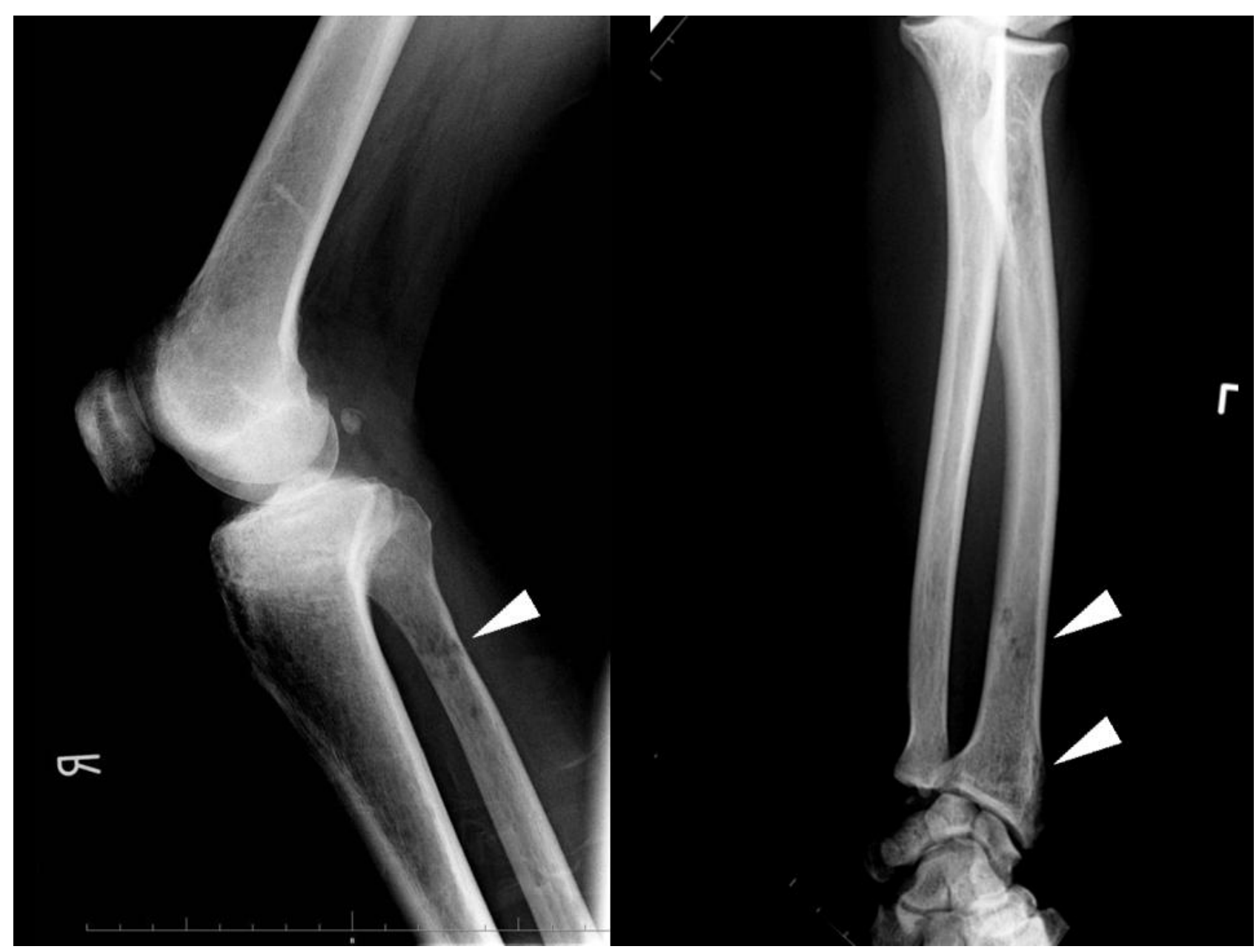


Figure B

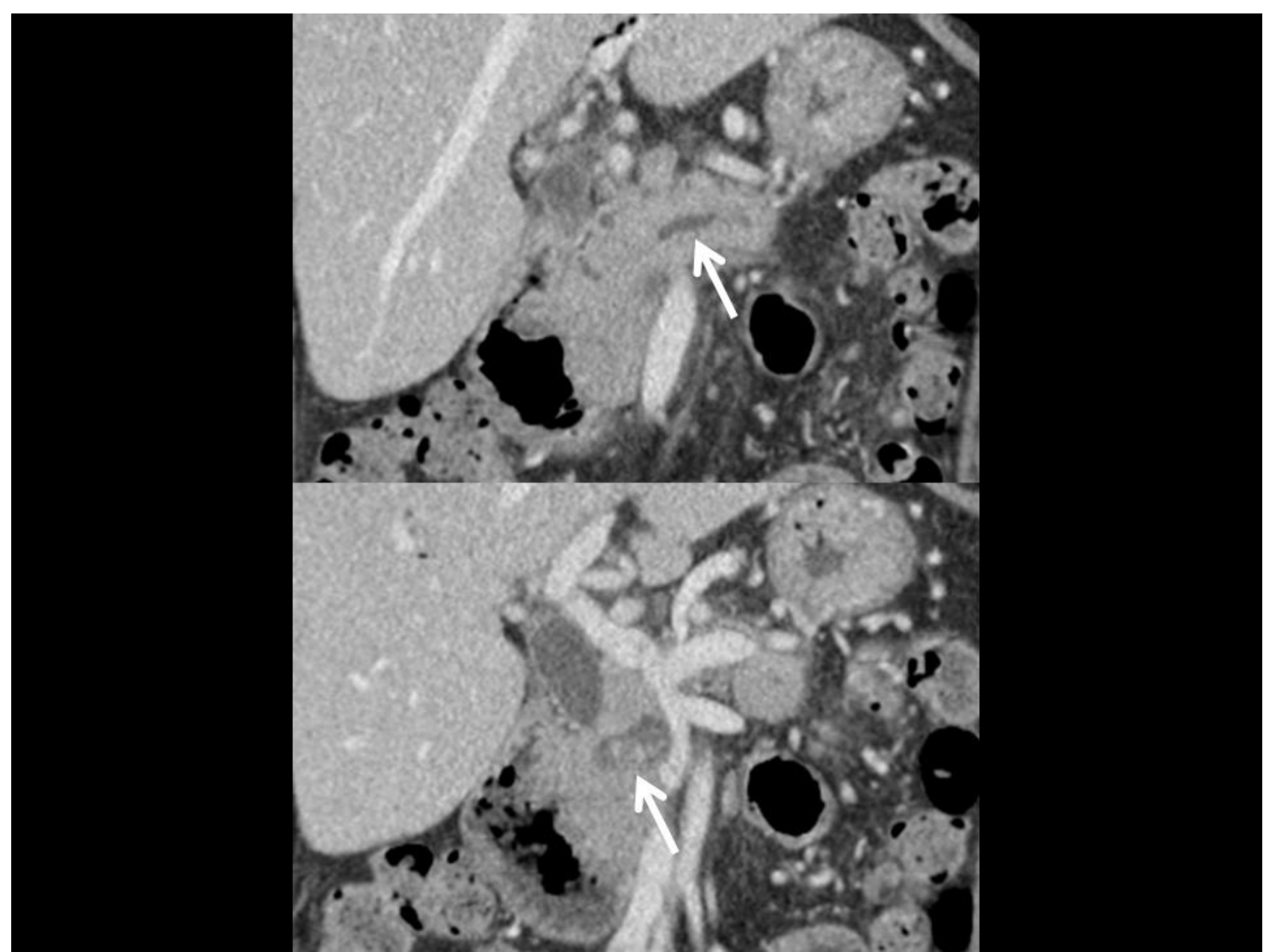


Figure C

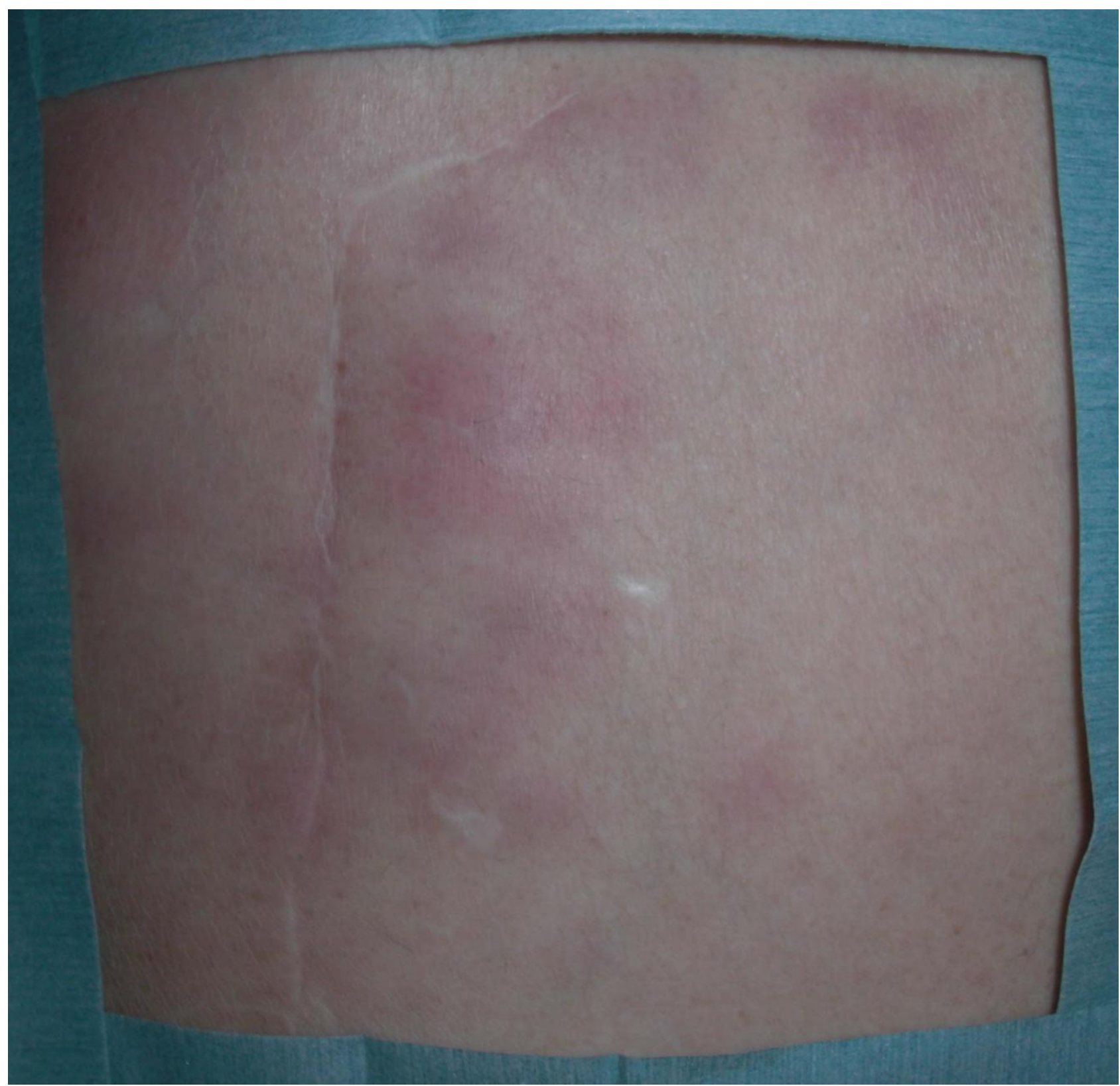


Figure D

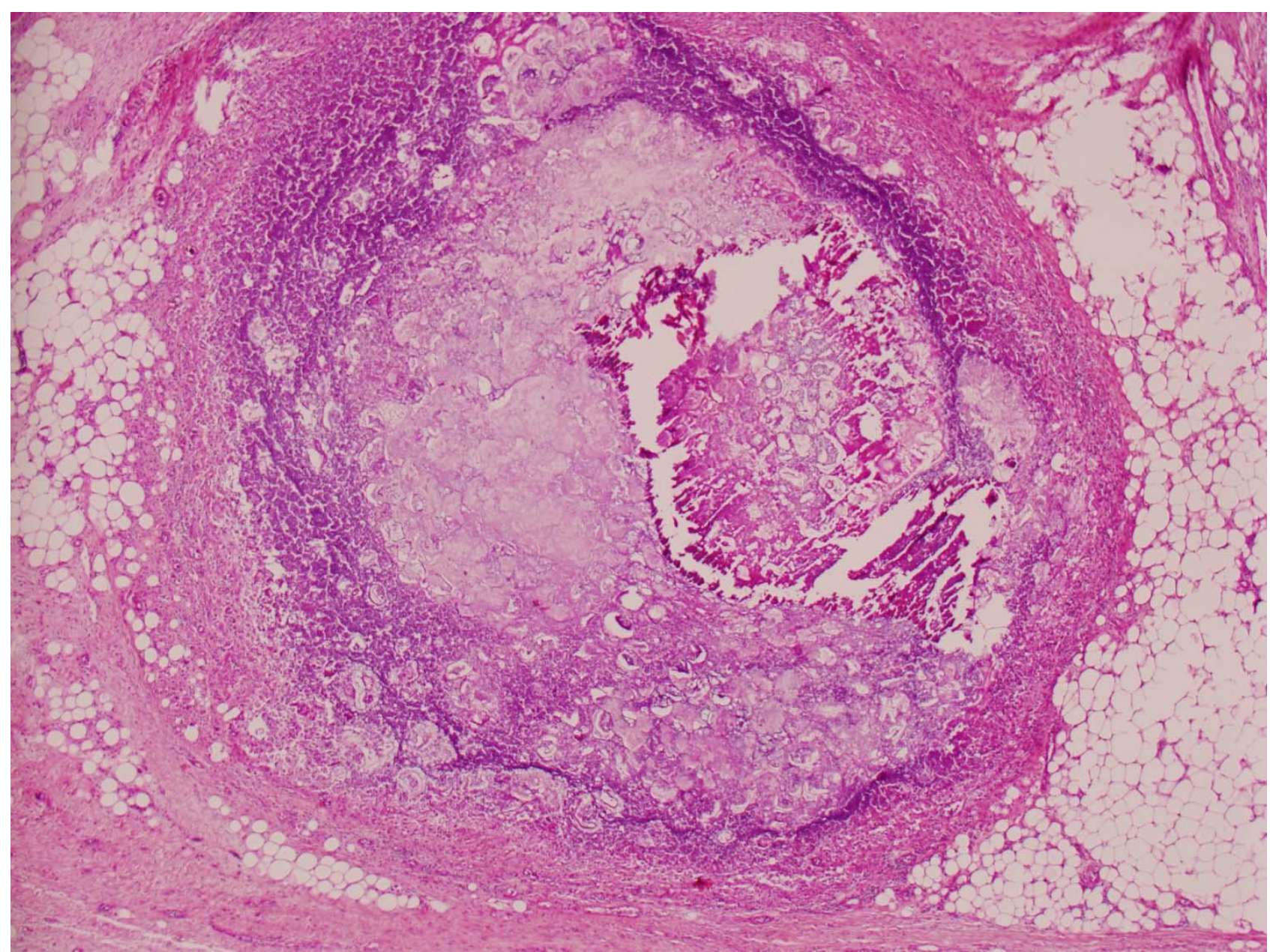

